\title{
Stress Detection Using Smartphone and Wearable Devices: A Review
}

\author{
Tejaswini Panure \\ School of Computer Science and \\ Engineering \\ Dr. Vishwanath Karad MIT World \\ Peace University \\ Pune, India \\ tejaswinipanure@gmail.com
}

\author{
Shilpa Sonawani \\ School of Computer Science and \\ Enigineering \\ Dr. Vishwanath Karad MIT World \\ Peace University \\ Pune, India \\ shilpa.sonawani@mitpune.edu.in
}

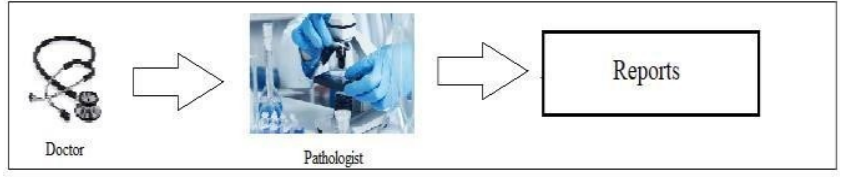

Abstract-Stress is the mental condition of the human body that causes it's dis-functioning. It affects adversely on body parts resulting in health disorders. Traditional method of stress detection includes lab tests done by doctor. Besides traditional techniques, sensors are used to measure physiological signals, as these signals make it easy to detect stress. Based on techniques of data collection, this paper is divided into two types, one for In-lab experiment, in which participants wear various sensors on their body which is invasive for real time application while in second, data was collected from sensors which are already available in the handy devices of participant such as smartphone, wearable devices etc. Different types of sensors and their uses are explained in this paper. Automatic real time stress detection systems can be developed. This paper lists various algorithms used to gain more accuracy in detecting stress. This paper is helpful for the fellow researchers who will be working on automatic stress detection. Various studies in this domain have been reviewed and this is a primary effort in summarizing the highlights of the previous research done in stress detection domain.

Keywords—Stress detection, Physiological signals, Fitbit, E4

\section{MOTIVATION}

According to World Health Organization (WHO), the count of people suffering from depression is nearly 350 million. Stress can lead to depression and further leads to suicide. Count of suicide is almost 1 million per year [31], so it is necessary to detect stress at primary level. Positive stress is like a motivation while negative stress badly affects human health as it is long lasting [34]. Being a long term it adversely affects human body, so it is necessary to detect negative stress. It is mostly seen in the people at working areas where the workload is more, along with deadlines and pressure of completion of the work.

\section{StRESS Detection System}

A stress detection methodologies studied here can be divided into three types as follows.
Figure 1. Traditional Stress Detection System 


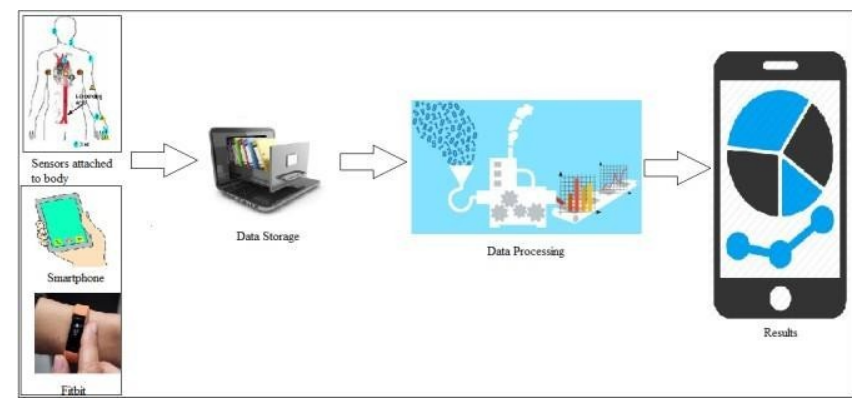

Figure 2. Automatic Stress Detection System

\section{A. Traditional Methods}

Following are few traditional methods to detect stress. Medical Tests - These can be done to know each physiological signals which reflects stress clearly for e.g. heart rate, galvanic skin response etc. [16], as shown in Figure 1.

Facial expression - In our day to day life, we come across many people. Some are known to us while some are not. We can easily detect the changes in behavior or the emotions of a person which are very well known to us. We can tell whether he/she is tensed by observing his/her behavior. But same thing doesn't happens with the person which are not known or very less known to us. Because

Few are explained in Table II below. By considering these two ways, let's start with stress detection using sensors.

\section{B. Using Sensors for Stress Detection}

When an individual is stressed, there are few fluctuations (depending on how much the person is stressed) in physiological parameter values [16]. Various physiological parameters used are explained in Table I. Sensors are attached to different body parts as shown in Figure 2. Autonomic nervous system manages various functionalities of a human body [16]. Sympathetic nervous system and parasympathetic nervous system are the categories of ANS. SNS controls the reactions occurred when person is stressed while PNS controls resting activities of body [17]. To calculate these parameters, various sensors are available. These physiological parameters are calculated using various sensors and used for stress detection as follows.

To record brainwaves, a device which is an interface between brain and computer is kept on head and later divided into different bandwidths [1].EEG (Electroencephalography) is used to record signals of brain activities. Neurofeedback technique, which is the modern way to reduce stress is also used. i-care stress is an android application in which data is taken in two ways, one from emotive device which records brain activities and other from questionnaire to know the state of stress. This raw data is processed, and stress level is calculated. To reduce the knowing someone's behavioral changes happens only when the person is known to you.

Hormone analysis - During stress, level of various hormones gets changed [33]. So by testing level of these hormones stress can be detected.

As stress is becoming one of the major risk factor to the health, it needs to be detected and reduced. To know whether the person is stressed or tensed, there should be some way that will detect their daily behavioral patterns and will detect changes in that patterns.

To know these patterns, system needs to collect required data of each user, analyze it and make inferences accordingly.

The main challenge in detecting stress is that it varies from person to person i.e. although same work is given to all, it shows different levels of stress for every other person [11]. Stress of a person can be detected by examining the changes in emotions, daily routine etc. But this cannot be applied for everyone. For this we need to collect all the data of an individual and reveal all the changes in the regular behavior. Collection of data can be done in two ways, one is using medical tests and other using sensors in those devices handled by the user. These devices include smartphones, smartwatches, Fitbit etc., which have many sensors in it,

calculated stress three actions are provided, user is free to choose one of them. Actions include concentration, music therapy, and relaxation. In concentration user has to control his/her mind, and by doing so, stress can be reduced. In relaxation, deep breathing technique is used, which makes user calm. Third technique is music therapy, which requires entire mind to work simultaneously, hence relaxes mind. Stress levels, before and after applying above actions are recorded and are shown graphically, so as the user can observe the changes.

TABLE I. PHYSIOLOGICAL PARAMETERS USED

\begin{tabular}{|c|c|}
\hline Physiological Signals/ parameters & References \\
\hline Electrodermal Activity of skin (EDA) & {$[17,18,20,22,25,29]$} \\
\hline Blood Volume Pulse (BVP) & {$[17]$} \\
\hline Electrocardiography (ECG) & {$[17,18]$} \\
\hline Electromyography (EMG) & {$[17,25]$} \\
\hline Heart Rate Variability (HRV) & {$[7]$} \\
\hline Heart Rate (HR) & {$[18,20,22]$} \\
\hline Respiration Rate (RR) & {$[20,25]$} \\
\hline Systolic Blood Pressure (SBP) & {$[20,29]$} \\
\hline Electroencephalography (EEG) & {$[26]$} \\
\hline
\end{tabular}

Sioni $\mathrm{R}$ discussed how different physiological signals are affected during stress and various techniques to measure these signals such as Electrodermal activity of skin, Blood Volume Pulse, Electrocardiography, Electromyography etc. To measure these, various sensors and equipment are required which needs to be kept on particular area of body. Also it is recommended that these sensors should be 
included in the wearable devices. Also, heart rate variability (HRV), is considered as one of the important factor deciding stress, as it is a non-stationary signal. Various operations like preprocessing, analysis, feature extraction are performed on ECG signals. Feature extraction is done on the basis of time domain analysis as well as frequency domain analysis [7].
TABLE II. VARIOUS ALGORITHMS USED

\begin{tabular}{|c|c|}
\hline Algorithms & References \\
\hline Random Forest & {$[11]$} \\
\hline Support Vector Machine (SVM) & {$[18,2,21,24,11]$} \\
\hline K-nearest Neighbor (KNN) & {$[18,2]$} \\
\hline j48 & {$[8,11]$} \\
\hline Naïve Bayes & {$[8,21,24]$} \\
\hline Linear discriminant function (LDF & {$[21]$} \\
\hline Bagging & {$[11]$} \\
\hline DBSCAN & {$[15]$} \\
\hline k-means & {$[15,26]$} \\
\hline
\end{tabular}

Feature extraction of Heart Rate, Galvanic Skin response, Heart Rate Variability is done using time and frequency as parameters, in which $92.75 \%$ accuracy is achieved. Knearest neighbor and Support Vector Machine algorithms are used for classification. Using different sensors, physiological data is taken from working people and dataset is generated (SWELL-KW dataset) [18].

To develop a real time system, neural networks are trained using signals like Respiration Rate, Heart Rate, and Systolic Blood Pressure. These proved most effective for accuracy [20]. The sensors capture data continuously resulting in more energy consumption, to avoid this, first data of galvanic skin response is taken and analyzed If it shows symptoms of stress then level of above mentioned physiological signals is taken. Stress detection process takes place only when these signals show the symptoms of stress. The study in which devices or machines are trained such that it recognizes human emotions and stimuli like artificial intelligence [5].

Instead of using various sensors to know stress, a single sensor i.e. Electro-dermal Activity sensor is used in [21]. For this, MIT Media lab 'stress database is used. Model is trained, tested and then used. $81.82 \%$ accuracy is achieved [21].

Using just two signals i.e. Heart Rate [22] and Galvanic Skin Response $[29,22]$ an accuracy of $99.5 \%$ is achieved. InLab experimentation is done. To collect data, sensors were wore by the participants [22].

Electroencephalography signals of a patient are continuously taken for medical diagnosis. EEG detects the change in brain activities which can be noticed by their frequency changes. These signals are used in $[6,26]$. Using k-means clustering [26], and deep learning methodology [6], patients are divided into different categories of stress. Just EEG signal is used, therefore consumption of human efforts and time is reduced. Dataset is obtained from Matrix Radiotherapy from Kolhapur [26].

\section{Automatic Sensing Methodologies}

Almost everyone uses smartphone frequently. It has various inbuilt sensors for different purposes as explained in Table

III. Less or more of these sensors are available in wearable devices like Fitbit, E4 etc. Uses of these sensors is explained in the 


\section{Asian Journal of Convergence in Technology ISSN NO: 2350-1146 I.F-5.11}

table. Also no special handling of these sensors is required. Pattern of using smartphone and sensing data impacts a lot on person's stress [14]. Along with this, sleeping pattern and social interaction is also taken for better results [4]. Data

But when smartphone is motionless (kept somewhere during sleep, meeting etc.) creates obstructions in collecting physical activity data. Same issue doesn't occur when wearable devices like smartwatches are used because same sensors are available in smartwatch also. Here smartphone and smartwatch are used for data collection [13].

Orientation and placement of these sensors in smartphone or other wearable devices greatly influence the success of this system. But in real time application the variability in the placement and orientation of these devices should be handled properly. Fusion of sensors is a technique in which data from sensors is analyzed together to get more accurate results [13]. According to Thapliyal H., using IoT, various devices can be developed for automatic stress detection. Various sensors that collect different types of data required to detect stress are explained. A mobile application is necessary to function these sensors and analyze data.

Here five wearable devices are taken for this study, these are HeartMath Inner Balance, Spire, WellBe, Zensorium Being, Zensorium Tinke. All these devices are attached with at least one, either android or iOS application having access to the location and calendar with the users permissions. According to this study, HRV method is important to detect stress.

TABLE III. SENSORS AND THEIR USES

\begin{tabular}{|c|c|}
\hline Sensors & Uses \\
\hline Accelerometer & $\begin{array}{l}\text { Gives Activity Data. } \\
\text { To detect activity of a person such as whether } \\
\text { he/she is stationary, walking or running [11] }\end{array}$ \\
\hline Microphone & $\begin{array}{c}\text { To detect audio surrounding of a person (silence, } \\
\text { voice or noise) [11] }\end{array}$ \\
\hline Conversation data & To detect if person is near conversation [11] \\
\hline $\begin{array}{l}\text { GPS data } \\
\text { (GPS Chip) }\end{array}$ & To know the location of person [11] \\
\hline Call log data & Information about time and duration of calls [11] \\
\hline Light sensor data & $\begin{array}{l}\text { If the phone is in dark, charging or locked for a } \\
\text { longer period of time(battery charging andlocking } \\
\text { data) [11] }\end{array}$ \\
\hline Optical Sensor & $\begin{array}{l}\text { Variations in blood volume and heart rate are } \\
\text { detected using LED light that reflects off tothe skin. } \\
\text { Available in Zensorium Being device [9]. }\end{array}$ \\
\hline $\begin{array}{l}\text { Infrared Blood } \\
\text { Flow Sensor }\end{array}$ & $\begin{array}{c}\text { Using IR light, blood flow speed in ear is calculated } \\
{[9] .}\end{array}$ \\
\hline $\begin{array}{c}\text { Heart Rate } \\
\text { Monitoring Sensors }\end{array}$ & Using Heart Rate Variability, heart rate is taken [9] \\
\hline
\end{tabular}

Gjoreski M., proved that using only smartphone creates many challenges. Though the work profile is same for the people, stress observed is different for each person. Here person wise different model worked better using random forest. Along with the use of sensors that detects physiological signals, voice

\section{Volume V Issue I}

obtained from these sensors is used for stress detection by various researchers, as explained below. Accelerometer sensor in smartphone collects physical activity of the user.

permission of user to his/her highly private and sensitive contents. Data from sensors of smartphone such as microphone, accelerometer, electronic compass, light sensor and app usage history is taken from user for this study. MoodExplorer is an android app which collects sensing information. This app sends notifications to user and reports their emotional state. Around 76\% accuracy is taken from ML model [14].

Jin Lu's combination of smartphone and Fitbit along with questionnaire data are used. In the beginning of this study, clinical diagnosis of each participant is done. From smartphone, location and physical activity of the user is taken. For clustering DBSCAN is used. Activity data, heart rate, sleeping patterns are extracted from Fitbit. Here multitask learning is a proposed method [15].

\section{DISCUSSION}

According to World Health Organization (WHO), in future one individual among four will be suffering from mental disorder [32]. As we saw in above studies, to know the person's stress, it is important to know all the activities of a user along with their physiological parameters. Data is captured in two different forms, one in constrained environment, taking few participants for particular period of time (having some restrictions) for the purpose of study and second in unconstrained environment i.e. participants were free to do anything without any restriction. jome studies captured this data using sensors. But to develop a ystem as a real time application, user will be in unconstrained nvironment.

Jow to capture data, few studies used special sensors which vere attached to the user's body, and some took data from the levices which user uses daily such as smartphone and various vearable devices.

But having these sensors attached, user's body created disturbance in their daily routine. So capturing data from devices which user uses daily will be more comfortable. Various algorithms and techniques are applied as shown in Table II.

analysis can also be done [11]. Person specific model works better [12], but for this, more labelled data is required and in case of general model, no labelling is required. Garcia-Ceja only accelerometer is used for sensing data.

Even for mood prediction, blood pressure, heart beats, blood pressure etc. can be considered. SMS, Email contents also gives basic idea of the mood of a person, but it requires 


\section{Asian Journal of Convergence in Technology ISSN NO: 2350-1146 I.F-5.11}

\section{CONCLUSION}

World Health Record of World Health Organization, mentioned that among four individuals, one will suffer from mental disorder. 450 million people around world are suffering from stress [32]. Although much of study is done in the area of stress detection, more accuracy in unconstrained environment is needed. As we have seen above, no extra handling of sensors will be preferred by a user. It is fruitful to take data using sensors which are already present in the devices handled by user frequently.

Physiological Signals and Fuzzy Logic. IEEE Transactions on Industrial Electronics, 58(10), pp.4857-4865.

[4] Kostopoulos, P., Kyritsis, A., Deriaz, M. and Konstantas, D. (2016). Stress Detection Using Smart Phone Data. Lecture Notes of the Institute for Computer Sciences, Social Informatics and Telecommunications Engineering, pp.340-351.

[5] Greene, S., Thapliyal, H. and Caban-Holt, A. (2016). A Survey of Affective Computing for Stress Detection: Evaluating technologies in stress detection for better health. IEEE Consumer Electronics Magazine, 5(4), pp.44-56.

[6] Liao, C., Chen, R. and Tai, S. (2018). Emotion stress detection using EEG signal and deep learning technologies. 2018 IEEE International Conference on Applied System Invention (ICASI).

[7] Ramteke, R. and Thool, V. (2017). Stress detection of students at academic level from heart rate variability. 2017 International Conference on Energy, Communication, Data Analytics and Soft Computing (ICECDS).

[8] Gjoreski, M., Luštrek, M., Gams, M. and Gjoreski, H. (2017). Monitoring stress with a wrist device using context. Journal of Biomedical Informatics, 73, pp.159-170.

[9] Thapliyal, H., Khalus, V. and Labrado, C. (2017). Stress Detection and Management: A Survey of Wearable Smart Health Devices. IEEE Consumer Electronics Magazine, 6(4), pp.64-69.

[10] Ollander, S., Godin, C., Campagne, A. and Charbonnier, S. (2016). A comparison of wearable and stationary sensors for stress detection. 2016 IEEE International Conference on Systems, Man, and Cybernetics (SMC).

[11] Gjoreski, M., Gjoreski, H., Lutrek, M. and Gams, M. (2015). Automatic Detection of Perceived Stress in Campus Students Using Smartphones. 2015 International Conference on Intelligent Environments.

[12] Garcia-Ceja, E., Osmani, V. and Mayora, O. (2016). Automatic Stress Detection in Working Environments From Smartphones' Accelerometer Data: A First Step. IEEE Journal of Biomedical and Health Informatics, 20(4), pp.1053-1060.

[13] Vaizman, Y., Ellis, K. and Lanckriet, G. (2017). Recognizing Detailed Human Context in the Wild from Smartphones and Smartwatches. IEEE Pervasive Computing, 16(4), pp.62-74.

[14] Zhang, X., Li, W., Chen, X. and Lu, S. (2018). MoodExplorer. Proceedings of the ACM on Interactive, Mobile, Wearable and Ubiquitous Technologies, 1(4), pp.1-30.

[15] Lu, J., Bi, J., Shang, C., Yue, C., Morillo, R., Ware, S., Kamath, J., Bamis, A., Russell, A. and Wang, B. (2018). Joint Modeling of Heterogeneous Sensing Data for Depression Assessment via Multi-task Learning. Proceedings of the ACM on Interactive, Mobile, Wearable and Ubiquitous Technologies, 2(1), pp.1-21.

[16] Xia, L., Malik, A. and Subhani, A. (2018). A physiological signal- based method for early mental-stress detection. Biomedical Signal Processing and Control, 46, pp.18-32.

[17] Sioni, R. and Chittaro, L. (2015). Stress Detection Using Physiological Sensors. Computer, 48(10), pp.26-33.

[18] Sriramprakash, S., Prasanna, V. and Murthy, O. (2017). Stress Detection in Working People. Procedia Computer Science, 115, pp.359- 366.

[19] Smith, A. and Thomas, A. (2018). Reducing the Consequences of Acute Stress on Memory Retrieval. Journal of Applied Research in Memory and Cognition, 7(2), pp.219-229.

\section{Volume V Issue I}

Combination of various sensors will result in better accuracy.

\section{REFERENCES}

[1] Mitrpanont J., Phandhu-fung, J., Klubdee, N., Ratanalaor, S., Pratiphakorn, P., Damrongvanakul, K., Piyawat Chuanvaree, P. and Mitrpanont, T. (2019). iCare-Stress: Caring system for stress - IEEE Conference Publication. [online] Ieeexplore.ieee.org. Available at: https://ieeexplore.ieee.org/document/8075319/ [Accessed 7 Jan. 2019].

[2] Akmandor, A. and Jha, N. (2017). Keep the Stress Away with SoDA: Stress Detection and Alleviation System. IEEE Transactions on Multi- Scale Computing Systems, 3(4), pp.269-282.

[3] de Santos Sierra, A., Sanchez Avila, C., Guerra Casanova, J. and Bailador del Pozo, G. (2011). A Stress-Detection System Based on

[20] Koussaifi, M., Habib, C. and Makhoul, A. (2018). Real-time stress evaluation using wireless body sensor networks. 2018 Wireless Days(WD).

21] Liu, Y. and Du, S. (2018). Psychological stress level detection based on electrodermal activity. Behavioural Brain Research, 341, pp.50-53.

[22] de Santos Sierra, A., Sanchez Avila, C., Guerra Casanova, J. and Bailador del Pozo, G. (2011). A Stress-Detection System Based on Physiological Signals and Fuzzy Logic. IEEE Transactions on Industrial Electronics, 58(10), pp.4857-4865.

23] Han, L., Zhang, Q., Chen, X., Zhan, Q., Yang, T. and Zhao, Z. (2017). Detecting work-related stress with a wearable device. Computers in Industry, 90, pp.42-49.

24] Zhang, X., Lyu, Y., Luo, X., Zhang, J., Yu, C., Yin, H. and Shi, Y. (2018). Touch Sense. Proceedings of the ACM on Interactive, Mobile, Wearable and Ubiquitous Technologies, 2(2), pp.1-18.

25] "Stress Detection Using Physiological Sensors", Riccardo Sioni and Luca Chittaro, Volume 48, Issue 10, October 2015.

[26] Kalas, M. and Momin, D. (2019). Stress detection and reduction using EEG signals - IEEE Conference Publication. [online] Ieeexplore.ieee.org. Available at: https://ieeexplore.ieee.org/document/7755604 [Accessed 8 Jan. 2019]. [27] Aigrain, J., Spodenkiewicz, M., Dubuiss, S., Detyniecki, M., Cohen, D. and Chetouani, M. (2018). Multimodal Stress Detection from Multiple Assessments. IEEE Transactions on Affective Computing, 9(4), pp.491-506.

28] VAIZMAN,, Y., WEIBEL, N. and LANCKRIET, G. (2019). Context Recognition In-the-Wild: Unified Model for Multi-Modal Sensors and Multi-Label Classification. [online] Extrasensory.ucsd.edu. Available at: http://extrasensory.ucsd.edu/papers/vaizman2017b imwutAcceptedVersion .pdf [Accessed 8 Jan. 2019]

[29] Fernandes, A., Helawar, R., Lokesh, R., Tari, T. and V. Shahapurkar, A. (2019). Determination of stress using Blood Pressure and Galvanic Skin Response - IEEE Conference Publication. [online] Ieeexplore.ieee.org. Available at: https://ieeexplore.iee.org/document/7062747 [Accessed 8 Jan. 2019]

30] Marina Marcus, M. Taghi Yasamy, Mark van Ommeren, and Dan Chisholm, Shekhar Saxena. (2012). Depression: A Global Public Health Concern | HESP News Briefing - RSS feeds. [Online]. Available: http://hespnews.org /2012 /10/ 05/depression-a-global-public-healthconcern/.

[31]http://www.who.int/mental health/management/depression/who_paper depression_wfmh_2012.pdf

[32]https://www.who.int/whr/2001/media centre/press release/en/

33] Ranabir, S. and Reetu, K. (2011). Stress and hormones. Indian Journal of Endocrinology and Metabolism, 15(1), p.18.

[34] https://www.uml.edu/docs/IntroductionToJobStress tcm18-42460.pdf 